\title{
Leigh Syndrome in an Adolescent Girl: A Case Report
}

\author{
Merina Shrestha, Luna Bajracharya, Sudha Basnet \\ Department of Child Health, Maharajgunj Medical Campus, Tribhuvan University Teaching Hospital, Institute of \\ Medicine, Maharajgunj, Kathmandu
}

\section{Corresponding author:}

Merina Shrestha, MBBS, MD

Department of Child Health, Maharajgunj Medical Campus, Tribhuvan University Teaching Hospital, Institute of Medicine, Maharajgunj, Kathmandu

Email: drmerinashrestha@gmail.com

Submitted : $21 \mathrm{Apr}, 2020$

Accepted : $9 \mathrm{Jul}, 2020$

\begin{abstract}
Leigh syndrome (LS) is a disorder of infancy and rarely late childhood. It presents with regression of mental and motor skills. Here, we present LS in an adolescent girl who presented with generalized dystonia and cognitive decline. Her infective, metabolic, endocrinal and autoimmune work up was normal. The neuroimaging showed progressive symmetric involvement of basal ganglia with focal intensity over bilateral lentiform nucleus and thalamic region. The cerebrospinal fluid (CSF) lactate level was elevated. Genetic test could not be performed; however the history of neuro-regression with extrapyramidal involvement with CSF and neuroimaging finding led to the diagnosis of Leigh's syndrome.
\end{abstract}

Keywords: neuro-metabolic, regression, MRI

\section{INTRODUCTION}

\section{L}

eigh syndrome (LS) was first described in 1952 by a British neuro-psychiatrist, Archibald Denis -Leigh. The condition is an inherited neurometabolic disease affecting the central nervous system. ${ }^{1}$ The prevalence of Leigh disease (LD) is about of $1: 100,000$ to $1: 140,000$ births. It is a familial or sporadic mitochondrial disorder characterized by psychomotor regression and lesions in the basal ganglia and brainstem. Onset of Leigh syndrome typically begins during infancy with progression and death by 2 years of age. ${ }^{2}$ Late onset disease during adolescence and adult is not common. ${ }^{3}$

\section{CASE PRESENTATION}

A 13-year girl presented with insidious progression of symptoms for 18 months. It started with gradual onset of tremor in the hands, slurring of speech and increased salivation. There was no history of fever, headache or vomiting at the onset of symptoms. For the past 8 months, she started to develop gradually progressive dystonia of upper limbs involving the entire body aggravated by stress or stimulus. Because of the dystonia, her daily activities were restricted but could walk around with support, eat by herself and take self care. For last one month, she developed motor aphasia along with swallowing difficulty. There was neither history of seizure or impairment of consciousness.

There was also no prior history of jaundice, joint pain, rash or abnormal behavior or altered sleep pattern or any history of head trauma. Other personal and family history was unremarkable. She was the only child and product of her non - consanguineous marriage.

On examination child was alert and oriented with generalized and oro-motor dystonia. Her vitals were normal. She had extrapyramidal signs along 
with exaggerated knee and ankle reflexes. Her vision and hearing was grossly intact at the time of admission; however detail auditory and visual assessment could not be performed. After about one week of hospital admission, she gradually deteriorated with increasing dystonia and cognitive decline. She started to become drowsy, failed to recognize parents and also developed urinary and bowel incontinence.

Her hemogram, renal and liver function tests were normal. Metabolic work up revealed normal serum copper, ceruloplasmin, and urine copper levels. Thyroid profile, anti-nuclear antibody (ANA), anti-double stranded DNA (anti-dsDNA) and serum lactate were also normal. Tandem mass spectroscopy including screening for abnormal urinary metabolites was also normal. Cerebrospinal fluid (CSF) analysis showed lymphocytes of 65 cells/dl with slight increase in protein level of $60 \mathrm{mg} /$ dl. CSF N-methyl-D-asparate (NMDA) receptor antibody was negative; however CSF lactate was increased. Her magnetic resonance imaging (MRI) of head showed hyper signal intensity over bilateral basal ganglia predominantly bilateral lentiform nucleus and thalamus; and electroencephalogram (EEG) showed generalized slowing with theta delta dysrhythmia.

She was started on supplements of thiamine, carnitine, pyridoxine, coenzyme-O and trihexyphenyldyl for her dystonia. During hospital stay, her dystonia decreased slightly however her cognition and incontinence remained static. With the available reports, the diagnosis of late onset Leigh disease was made.

\section{DISCUSSION}

Leigh disease is considered as a disease in infancy or early childhood. Defects in mitochondrial oxidative phosphorylation (OXPHOS) is usually associated with early onset disease. ${ }^{4}$ It can be inherited as an autosomal recessive mitochondrial trait due to mutations in nuclear genes encoding mitochondrial respiratory chain complex subunits or complex assembly proteins. ${ }^{5} \mathrm{X}$-linked related to defects in pyruvate dehydrogenase complex (PDHc) due to mutations in the PDHA1 gene is also found to be associated with LD. ${ }^{6}$

In young infants, LD presents with motor delay, mental retardation and/or progressive cognitive decline, hypotonia, ataxia and dystonia. Because of involvement of brainstem, the children might present with respiratory abnormalities and swallowing dysfunction along with ophthalmological manifestations and abnormal thermoregulation. ${ }^{7}$

The late onset LD is not common. In older children and adults, it presents with cranial nerve disturbance, pyramidal signs, and cerebellar dysfunction.
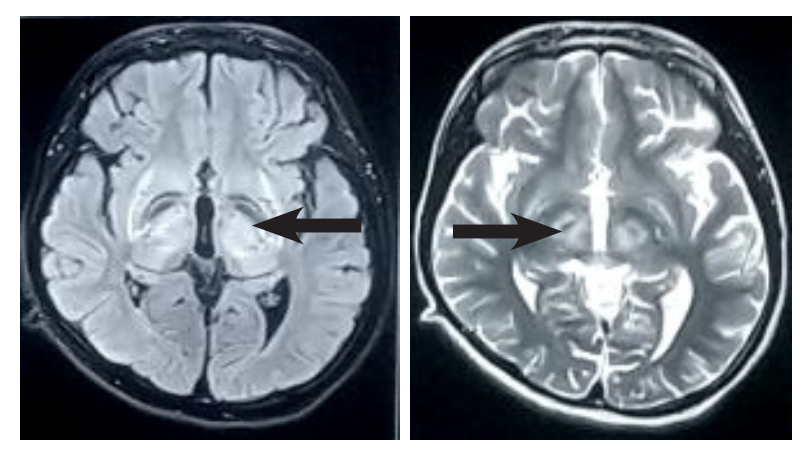

Figure 1a. Flair picture MRI. Figure 1b. T2 weighted MRI show hyper signal intensity over bilateral basal ganglia predominantly bilateral lentiform nucleus and thalamus.

Rahman et al. proposed the diagnostic criteria for LS and are as follows: ${ }^{8}$

1. Progressive neurologic disease with motor and intellectual developmental delay;

2. Signs and symptoms of brain stem and/or basal ganglia dysfunction;

3. Elevated lactate levels in the blood and/or CSF; and

4. One or more of the following:

a. Characteristic features of LS on neuroimaging (symmetric hyperintense lesions in basal ganglia and/or brainstem in T2 sequence)

b. Typical neuropathological changes at postmortem examination, and

c. Typical neuropathology in a similarly affected sibling.

Another criteria to diagnose LD in adults proposed by Sakushima et al. as follows : ${ }^{9}$

1. History of cryptogenic thrive failure or signs of mental retardation, pyramidal signs, cerebellar disturbances, ophthalmoplegia, deafness, dysarthria, or other neurological symptoms are present

2. Bilateral basal ganglia lesions or brainstem lesions with serum or CSF lactate elevation are present (lactate stress test (LST) should be considered when resting lactate levels are normal)

3. Mitochondrial abnormalities are present in muscle pathology or in biochemical analyses, or known LD gene mutations are present

4. Metabolic disorders, toxins, infection, multiple sclerosis, and Wernicke's encephalopathy can be excluded.

Our patient fulfilled most of the Rahman's criteria along with striking MRI finding except for post mortem and genetic test. Wilson disease, infections, thiamine deficiency, multiple sclerosis and other 
metabolic diseases were differential diagnosis for the patient and were excluded by investigations. During her hospital stay of almost 4 weeks, we treated her with pulse dose of methylprednisolone for five days. Mega dose of thiamine and supplementation of carnitine, pyridoxine and coenzyme-Q was continued. However, she did not respond to any of the treatment. Her cognition and consciousness level gradually started to deteriorate. She was then discharged with above medications on parents request.

The prognosis of $L D$ is brim and there is no definitive treatment for LD till now. Poor survival is predicted by disease onset before 6 months of age, failure to thrive, brainstem lesions on neuroimaging and requirement for intensive care treatment. The major cause of mortality is due to disease progression with progressive brain stem involvement with respiratory insufficiencies. ${ }^{10}$

\section{CONCLUSION}

Leigh disease should be considered in an adolescent if they present with progressive extrapyramidal symptoms and neuroimaging findings of bilateral symmetric involvement of multiple brainstem nuclei/ structures. In such condition, further investigations including blood and/or CSF lactate, respiratory chain enzymes activities and genetic studies should be considered to support the diagnosis. Though the complete cure for LD is not available, accurate diagnosis with appropriate investigations followed by prompt institution of adequate supportive therapy helps in amelioration of apparent symptoms as well parental dilemma of the disease.

\section{CONFLICT OF INTEREST}

None declared.

\section{CONSENT}

Consent was obtained from parents to publish this report and share the MRI images.

\section{REFERENCES}

1. Noble P. Denis Archibald Leigh. Psychiatric Bulletin. 1998;22(10):648-649.

2. Genetics Home Reference. Leigh Syndrome. National Institute of Health. 2013.

3. Huntsman RJ, Sinclair D B, Bhargava R et al. Atypical Presentations of Leigh Syndrome: A Case Series and Review. Pediatric Neurology. 2005;32(5):334-340

4. Darin N, Oldfors A, Moslemi AR et al. The Incidence of Mitochondrial Encephalomyopathies in Childhood: Clinical Features and Morphological, Biochemical, and DNA Abnormalities. Annals Of Neurology. 2001;49(3):377-383.

5. Moslemi AR, Darin N. Molecular Genetic and Clinical Aspects of Mitochondrial Disorders in Childhood. Mitochondrion. 2007;7(4):241-252.

6. Matthew $P$, Marchington D, Squier M et al. Molecular Genetic Characterization of an X-Linked Form of Leigh's Syndrome. Annals of Neurology. 1993;33(6):652-655.

7. Montpetit VJ, Andermann F, Carpenter S et al. Subacute Necrotizing Encephalomyelopathy: A Review and a Study of Two Families. Brain. 1971;94(1):1-30.

8. Rahman S, Blok R, Dahl HH et al. Leigh Syndrome: Clinical Features and Biochemical and DNA Abnormalities. Annals of Neurology. 1996;39(3):343-351

9. Sakushima K. Tsuji-Akimoto S, Niino M et al. Adult Leigh Disease Without Failure to Thrive. The Neurologist. 2011;17(4):222-227.

10. Sofou K, De Coo IF, Isohanni P et al. A Multicenter Study on Leigh Syndrome: Disease Course and Predictors of Survival. Orphanet Journal of Rare Diseases. 2014;9(1):52. 